\title{
Cinnamaldehyde, Cinnamic Acid, and Cinnamyl Alcohol, the Bioactives of Cinnamomum cassia Exhibit HDAC8 Inhibitory Activity: An In vitro and In silico Study
}

Mangesh Patil, Amit S. Choudhari', Savita Pandita', Md Ataul Islam², Prerna Raina', Ruchika Kaul-Ghanekar'

Computer Applications Department, NBN Sinhagad Technical Institutes Campus, NBN Sinhagad School of Computer Studies, 'Cancer Research Lab, Interactive Research School for Health Affairs, Bharati Vidyapeeth Deemed University, Pune-Satara Road, Pune, Maharashtra, India, ${ }^{2}$ Department of Chemical Pathology, Faculty of Health Sciences, Tshwane Academic Division of the National Health Laboratory Service, University of Pretoria, Pretoria, South Africa

\begin{abstract}
Background: The altered expression of histone deacetylase family member 8 (HDAC8) has been found to be linked with various cancers, thereby making its selective inhibition a potential strategy in cancer therapy. Recently, plant secondary metabolites, particularly phenolic compounds, have been shown to possess HDAC inhibitory activity. Objective: In the present work, we have evaluated the ability of cinnamaldehyde (CAL), cinnamic acid (CA), and cinnamyl alcohol (CALC) (bioactives of Cinnamomum) as well as aqueous cinnamon extract (ACE), to inhibit HDAC8 activity in vitro and in silico. Materials and Methods: HDAC8 inhibitory activity of ACE and cinnamon bioactives was determined in vitro using HDAC8 inhibitor screening kit. Trichostatin A (TSA), a well-known anti-cancer agent and HDAC inhibitor, was used as a positive control. In silico studies included molecular descriptor Analysis molecular docking absorption, distribution, metabolism, excretion, and toxicity prediction, density function theory calculation and synthetic accessibility program. Results: Pharmacoinformatics studies implicated that ACE and its Bioactives (CAL, CA, and CALC) exhibited comparable activity with that of TSA. The highest occupied molecular orbitals and lowest unoccupied molecular orbitals along with binding energy of cinnamon bioactives were comparable with that of TSA. Molecular docking results suggested that all the ligands maintained two hydrogen bond interactions within the active site of HDAC8. Finally, the synthetic accessibility values showed that cinnamon bioactives were easy to synthesize compared to TSA. Conclusion: It was evident from both the experimental and computational data that cinnamon bioactives exhibited significant HDAC8 inhibitory activity, thereby suggesting their potential therapeutic implications against cancer.

Key words: Absorption, cinnamon, density function theory, distribution, excretion, HDAC8, metabolism, toxicity prediction, molecular docking, synthetic accessibility

\section{SUMMARY}

- Pharmacoinformatics studies revealed that cinnamon bioactives bound to the active site of HDAC8 enzyme in a way similar to that of TSA

- The molecular descriptors of cinnamon compounds successfully correlated with TSA values. The binding interactions and energies were also found to be close to TSA

- Synthetic accessibility values showed that cinnamon bioactives were easy to synthesize compared to TSA.
\end{abstract}

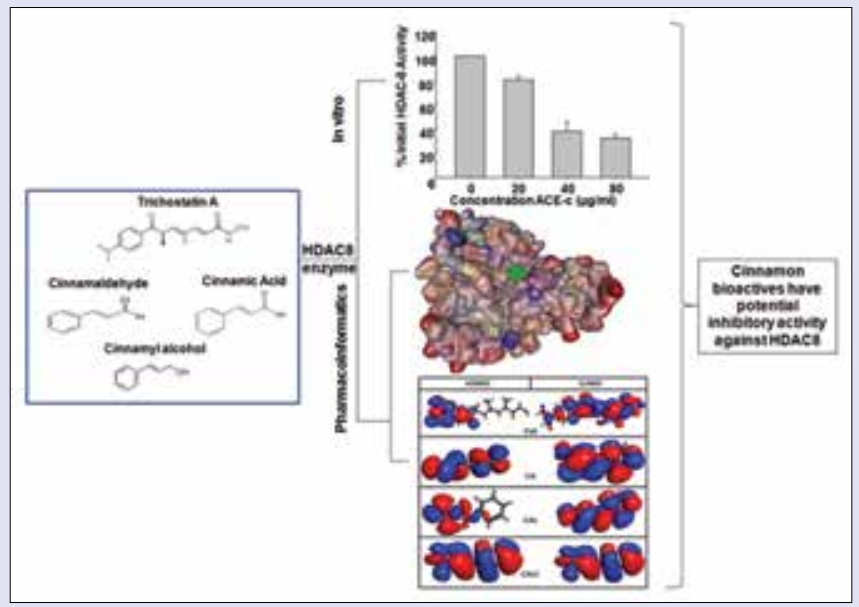

Abbreviations used: ACE: Aqueous Cinnamon Extract; DFT: Density Function Theory; CAL: Cinnamaldehyde; CA: Cinnamic Acid; CALC: Cinnamyl Alcohol; MW: Molecular Weight; ROTBs: Rotatable Bonds; ROF: Lipinski's Rule of Five; TSA: Trichostatin A; PDB: Protein Data Bank; RMSD: Root Mean Square Deviation; HBA: Hydrogen Bond Acceptor; HBD: Hydrogen Bond Donor; ADMET: Absorption, Distribution, Metabolism, Excretion and Toxicity; FO: Frontier Orbital; HOMOs: Highest Occupied Molecular Orbitals; LUMOs: Lowest Unoccupied Molecular Orbitals; BE: Binding Energy.

Correspondence:

Dr. Ruchika Kaul-Ghanekar, Interactive Research School for Health Affairs, Bharati Vidyapeeth University Medical College Campus, Katraj-Dhankawadi, Pune - 411 043, Maharashtra, India.

E-mail: ruchika.kaulghanekar@gmail.com DOI: 10.4103/pm.pm_389_16

\section{Access this article online} Website: www.phcog.com

Quick Response Code:

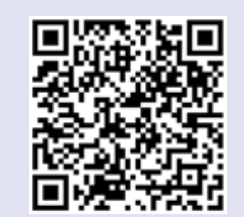

\section{INTRODUCTION}

Histone deacetylases (HDACs) are enzymes that have specific role in development and tissue homeostasis..$^{[1]}$ Based on phylogenetic similarity, HDACs have been categorized into four classes, Class I (HDAC1, 2, 3, and 8); II (HDAC4, 5, 6, 7, 9, and 10); and IV (HDAC11). ${ }^{[2]}$ Biochemical analysis has revealed that Class I HDACs hold majority of HDAC activity, whereas purified recombinant Class II HDACs possess only minimal activity. ${ }^{[3-5]}$ In the recent years, HDACs have emerged as important therapeutic targets for various diseases, such as cancer, cardiovascular
This is an open access article distributed under the terms of the Creative Commons Attribution-NonCommercial-ShareAlike 3.0 License, which allows others to remix, tweak, and build upon the work non-commercially, as long as the author is credited and the new creations are licensed under the identical terms.

\section{For reprints contact: reprints@ medknow.com}

Cite this article as: Patil M, Choudhari AS, Pandita S, Islam M, Raina P, Kaul-Ghanekar R. Cinnamaldehyde, Cinnamic Acid, and Cinnamyl Alcohol, the Bioactives of Cinnamomum cassia Exhibit HDAC8 Inhibitory Activity: An In vitro and In silico Study. Pharmacogn Mag 2017; 13(51): 645-651. 
diseases, autoimmunity, and neurodegenerative diseases. ${ }^{[6-9]}$ In cancer, deregulation of HDAC activity due to aberrant expression or recruitment to promoter regions results into uncontrolled growth. ${ }^{[2,10]}$ HDAC family member 8 (HDAC8) has been found to play a crucial role in the physiology of both hematological and solid malignancies. ${ }^{[1]}$ Knockdown studies of HDAC8 have been shown to alter the growth of human lung, colon, and cervical cancer cells, ${ }^{[12]}$ and induce cell cycle arrest as well as differentiation of neuroblastoma cells. ${ }^{[13]}$

HDAC inhibitors have emerged as potent anticancer agents that can restore programmed cell death in malignant cells..$^{[14,15]}$ Compared to other Class I isoforms, HDAC8 could be an attractive target due to the presence of unique second metal binding site in proximity to the main catalytic domain, which displays a therapeutic potential with greater efficacy for selective inhibition. ${ }^{[16]}$ Recently, there has been growing interest in plant secondary metabolites, particularly phenolic compounds that possess HDAC inhibitory activity. ${ }^{[17]}$ Many studies have indicated that cancer prevalence could be significantly modulated by an increased intake of diet rich in polyphenols such as fruits and vegetables. ${ }^{[18-20]}$ Various polyphenols such as green tea catechin (-)-epigallocatechin-3-gallate, curcumin, resveratrol, soy isoflavones, quercetin, and caffeic acid have been reported to exhibit HDAC inhibitory potential. ${ }^{[20-22]} \mathrm{A}$ few studies have demonstrated synergistic activity of plant-based histone modifiers, when combined with ionizing irradiation or DNA-damaging drugs. ${ }^{[21]}$

Cinnamon, a commonly used food spice, contains several bioactive components such as cinnamic acid, cinnamyl aldehyde, tannin, mucus, and carbohydrates. ${ }^{[22]}$ Cinnamon has been known to exhibit various pharmacological activities including antioxidant, ${ }^{[23-26]}$ anti-inflammatory, ${ }^{[27,28]}$ antimicrobial, ${ }^{[29-32]}$ antipyretic, ${ }^{[33]}$ antiulcer, ${ }^{[34,35]}$ antidiabetic, ${ }^{[36-40]}$ and antitumor. ${ }^{[41,42]}$ We have previously reported chemopreventive potential of aqueous cinnamon extract (ACE) in cervical cancer, wherein it induced apoptosis in $\mathrm{SiHa}$ cell line through loss of mitochondrial membrane potential. ${ }^{[41]}$ To evaluate whether the apoptosis induced by ACE was due to inhibition of HDAC8, we examined its activity in a cell-free system. Further, various pharmacoinformatics techniques such as structure activity relationship ${ }^{[43,44]}$ molecular docking, ${ }^{[45]}$ density function theory (DFT), ${ }^{[4]}$ toxicity prediction, ${ }^{[47]}$ and synthetic accessibility ${ }^{[47]}$ were adopted to elucidate HDAC8 inhibitory potential of the test materials.

\section{MATERIALS AND METHODS}

\section{Preparation of extract and determination of its histone deacetylase inhibitory activity}

The bark of Cinnamomum cassia was purchased from Shivam Ayurvedics, Pune, Maharashtra, India, with voucher specimen number 104. The sample was authenticated from Regional Research Institute (AY) Kothrud, Pune (ref no. 1045). The ACE was prepared as described earlier, ${ }^{[41]}$ and HDAC8 inhibitor screening assay was performed as per the manufacturer's instructions (Cayman chemical, USA). Briefly, the reaction was initiated in a 96-well plate which contained $25 \mu$ l assay buffer, $5 \mu$ l HDAC 8 enzyme, $5 \mu \mathrm{l}$ of extract/inhibitor at various concentrations $(0-80 \mu \mathrm{g}), 15 \mu \mathrm{l}$ substrate, i.e., Arg-His-Lys-Lys ( $\varepsilon$-acetyl)-AMC p53 sequence $(100 \mu \mathrm{M})$ and incubated at $37^{\circ} \mathrm{C}$ for $30 \mathrm{~min}$. Following the incubation, $50 \mu \mathrm{l}$ of developer/stop solution was added, and the fluorescence was analyzed with an excitation wavelength of 350-360 $\mathrm{nm}$ and an emission wavelength of 450-465 nm using a microplate reader (BMG, Fluostar Omega). Percentage inhibition activity was calculated by the formula: $\%$ inhibition $=([$ Initial activity - inhibitor $] /$ initial activity $) \times 100$.

\section{RESULTS AND DISCUSSION}

Effect of aqueous cinnamon extract and its

bioactives (cinnamaldehyde, cinnamic acid, cinnamyl alcohol) on histone deacetylases family member 8 inhibitory activity

HDAC8 activity was significantly inhibited at $40(\sim 62 \%)$ and 80 $\mu \mathrm{g} / \mathrm{ml} \quad(\sim 67 \%)$ concentrations of ACE. $\mathrm{IC}_{50}$ value of ACE was $25.24 \mu \mathrm{g} / \mathrm{ml}$ [Figure 1]. We further analyzed whether ACE and its bioactives (cinnamaldehyde [CAL], cinnamic acid [CA], cinnamyl alcohol [CALC]) exhibited HDAC8 inhibitory activity. Before this, we performed high-performance liquid chromatography analysis of ACE to confirm cinnamon bark identity by detecting the presence of marker molecules, CAL, CA, and CALC have been provided in Figure 2. This was conducted using a Phenomenex C18 $(4.6 \mathrm{~mm} \times 250 \mathrm{~mm}, 5 \mu \mathrm{m}$; Phenomenex, Torrance, CA, USA) column whose temperature was set at $40^{\circ} \mathrm{C}$. Gradient flows for the two solvent systems (solvent $\mathrm{A}, 0.1 \%$ phosphoric acid in water; solvent $\mathrm{B}$, acetonitrile) were: $0 \mathrm{~min}, 10 \% \mathrm{~B}$; $12 \mathrm{~min}, 20 \% \mathrm{~B} ; 35 \mathrm{~min}, 50 \% \mathrm{~B} ; 40 \mathrm{~min}$, and $100 \% \mathrm{~B}$ and hold at $100 \% \mathrm{~B}$ for $5 \mathrm{~min}$. The standard marker compounds, CAL, CA, and CALC, were used. The flow rate of the mobile phase was $1.0 \mathrm{ml} / \mathrm{min}$. The injection volume was $10 \mu \mathrm{l}$, and the chromatogram was monitored at a wavelength of $265 \mathrm{~nm}$ throughout the experiment. It was observed that $80 \mu \mathrm{g} / \mathrm{ml}$ of ACE contained $310.25,60.75$, and $24.2 \mu \mathrm{M}$ concentrations of CAL, CA, and CALC, respectively [Figure 2]. At these particular concentrations, CAL, CA, and CALC showed 18.5, 9.3, and 5.7\% inhibition of HDAC8 activity, respectively [Table 1 ].

Moreover, combination of three (CAL + CA + CALC) inhibited HDAC8 activity by $37.7 \%$ [Table 1]. Thus, compared to the whole ACE, the combination (CAL + CA + CALC) contributed to almost half of HDAC8 inhibition. These results suggested that besides the selected bioactives of cinnamon, there may be other compounds in the extract that might have contributed to HDAC8 inhibition.

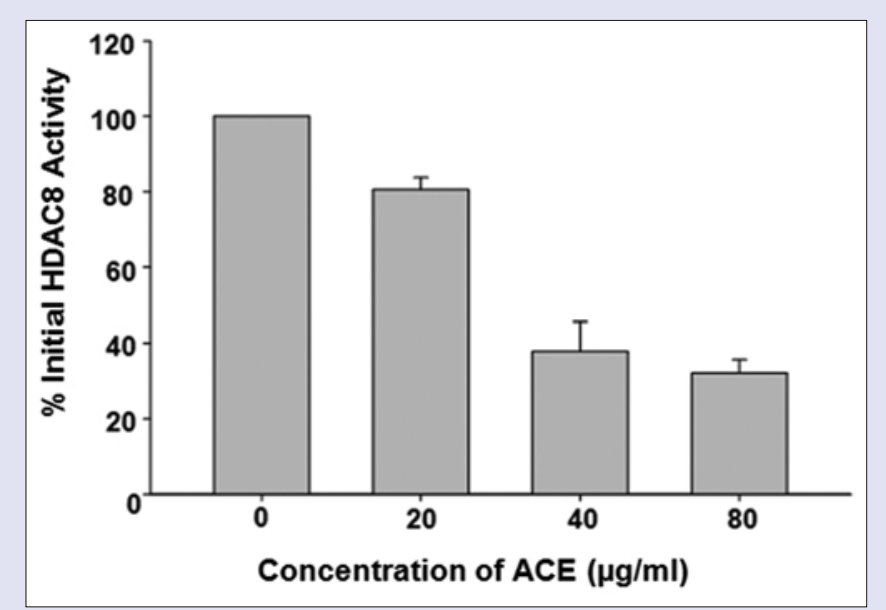

Figure 1: HDAC8 inhibition by aqueous cinnamon extract in a cell free system. ACE was examined for its HDAC8 inhibitory activity in a cell free system at concentrations of $20-80 \mu \mathrm{g} / \mathrm{ml}$. The results have been expressed as mean \pm standard deviation of three independent experiments 
Table 1: HDAC8 inhibition by aqueous cinnamon extract and its bioactives

\begin{tabular}{lccc}
\hline Test material & Concentration $(\mu \mathrm{g} / \mathrm{ml})(\%)$ & Concentration $(\mu \mathrm{M} / \mathrm{ml})$ & HDAC8 inhibitory activity $(\%)$ \\
\hline ACE & $80.00(100)^{*}$ & - & 67 \\
CAL & $40.69(50.08)$ & 310.25 & 18.5 \\
CA & $9.00(11.25)$ & 60.75 & 9.3 \\
CALC & $3.96(4)$ & 24.2 & 5.7 \\
Combination (CAL + CA + CALC) & $53.65(67.06)$ & $310.25+60.75+24.2$ & 37.7 \\
\hline
\end{tabular}

${ }^{*}$ Assuming $80 \mu \mathrm{g}=100 \%$. HDAC8: Histone deacetylase family member 8; CAL: Cinnamaldehyde; CA: Cinnamic acid; ACE: Aqueous cinnamon extract; CALC: Cinnamyl alcohol

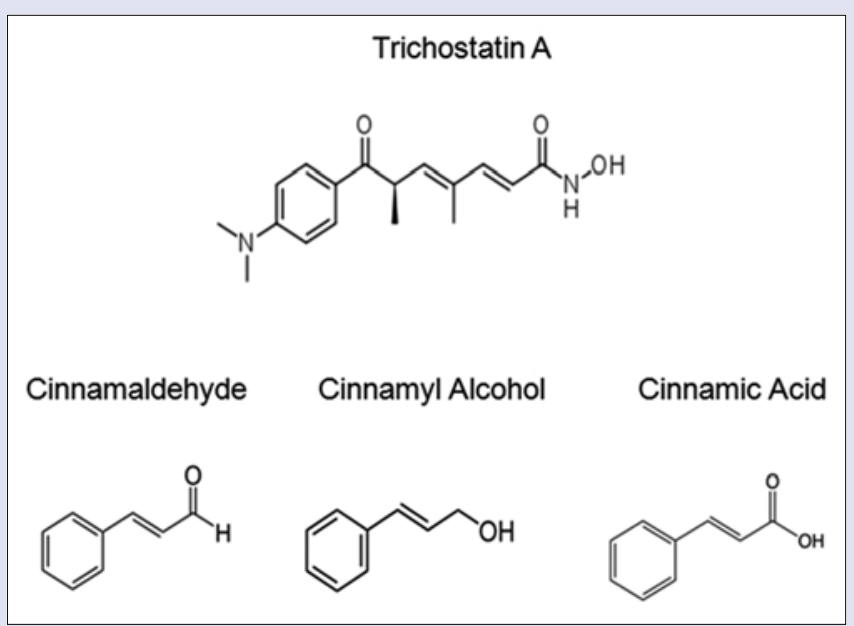

Figure 2: Chemical structures of trichostatin A, cinnamaldehyde, cinnamyl alcohol, and cinnamic acid

\section{Descriptor analysis, absorption, distribution,} metabolism, excretion, and toxicity, density function theory, molecular docking, and synthetic accessibility studies of aqueous cinnamon extract, cinnamaldehyde, cinnamic acid and cinnamyl

\section{alcohol, and trichostatin A}

Descriptor analysis included physicochemical properties, molecular weight (MW), number of rotatable bonds (ROTBs), molecular volume, polar surface area, and bioavailability score. The drug likeness was also analyzed by Lipinski's Rule of Five (ROF). To understand how the ligands (CAL, CALC, and CA along with trichostatin A [TSA]) bind to HDAC8 enzyme, molecular docking was carried out using AutoDock 4.2 tool, Molecular Graphics Lab, Scrrips Research Institute, California tool that uses the Lamarckian Genetic algorithm. ${ }^{[14,59]} 3 \mathrm{D}$ crystal structure of HDAC8 protein (Protein Data Bank [PDB] ID: 1T67) ${ }^{[14]}$ was collected from RCSB-PDB based on bound ligand, date of deposition, and resolution. For molecular docking, twenty conformations of each ligand were generated, and best conformer was considered for further study. The grid preparation was carried out based on the information of the bound ligand B3N (4-(dimethylamino)-N-[7-(hydroxyamino)-7-oxoheptyl] benzamide) at the active site of 1T67. ${ }^{[14]}$ From the docking results, the best docked pose was checked to evaluate whether it was able to form potential hydrogen-bonding interactions with the critical amino residues. Further, root mean square deviation (RMSD) between the best pose and the co-crystal was calculated by superimposing to govern whether the parameters used in the docking were able to replicate a conformation similar to that of the co-crystal at the active site cavity. The ligands were docked using similar parameters that were used for the co-crystal docking. Drug-like descriptors were calculated to evaluate
Table 2: Molecular descriptors of trichostatin A, cinnamaldehyde, cinnamic acid, and cinnamyl alcohol

\begin{tabular}{lcccc}
\hline Descriptors & TSA & CAL & CA & CALC \\
\hline $\log P$ & 2.683 & 2.484 & 1.91 & 2.032 \\
MW & 302.374 & 132.162 & 148.161 & 134.178 \\
TPSA & 69.635 & 17.071 & 37.299 & 20.228 \\
Number of atoms & 22 & 10 & 11 & 10 \\
Number of ON & 5 & 1 & 2 & 1 \\
Number of OHNH & 2 & 0 & 1 & 1 \\
Number of violations of ROF & 0 & 0 & 0 & 0 \\
ROTBs & 6 & 2 & 2 & 2 \\
Volume & 293.12 & 130.444 & 138.462 & 136.28 \\
Binding energy & -7.77 & -5.27 & -5.14 & -5.8 \\
Bioactivity score & 0.63 & -0.46 & -0.3 & -0.24 \\
\hline
\end{tabular}

CAL: Cinnamaldehyde; CA: Cinnamic acid; ACE: Aqueous cinnamon extract; CALC: Cinnamyl alcohol; MW: Molecular weight; ROF: Rule of five; ROTBs: Rotatable bonds; TPSA: Total polar surface area

drug likeness and to determine whether the bioactives of cinnamon exhibited properties similar to HDAC8 inhibitors. MW, $\log P$ value, hydrogen bond acceptor (HBA), and hydrogen bond donor (HBD) for each ligand were generated using molinspiration [Table 2].

Except for MW, volume, and surface area, rest of the descriptors showed comparable values to TSA. This indicated that all the three bioactives had induced HDAC8 inhibition. The hydrophobicity $(\log P)$ of TSA was 2.683 and that of CAL, CA, and CALC was found to be 2.484, 1.910, and 2.032, respectively. There was no violation of ROF for any compounds. ROF rules ${ }^{[51]}$ have been applied for the majority of molecules with good oral absorption and include that $\log P$ should be $<5$; MW should not be more than $500 \mathrm{kDa}$; number of HBD should be $<5$; and number of HBA should be $<10$. Number of ROTBs was found to be two for all the compounds of interest, while it was 6 for TSA. The data for all the descriptors were found to be within the proposed limit, thereby confirming their drug likeness.

Higher the bioactivity score of a compound, greater would be its probability to be active. In general, a compound with bioactivity score $>0.00$ would have considerable biological activity; with values between -0.50 and 0.00 , the compound could be moderately active; and with score below -0.50 , the compound could be inactive. ${ }^{[50]}$ Bioactivities of all the test compounds have been depicted in Table 2. TSA, a known enzyme inhibitor, showed highest (0.63) bioactivity score, whereas CALC, CA, and CAL showed score of $-0.46,-0.3$, and -0.24 , respectively. This indicated that all the compounds were moderately active and may possess enzyme inhibition activity.

Molecular docking is one of the crucial techniques in drug design and validation process. It gives accurate and preferred orientations of the ligand at the active site of the enzyme. ${ }^{[52,53]}$ The docked complexes of CAL, CA, and CALC were assessed for their optimal orientation and binding abilities. The crystal structure of HDAC8 (PDB ID: 1T67) was collected from RCSB-PDB. ${ }^{[50]}$ In the present study, self-docking approach was considered to validate the docking procedure, and the freshly docked complex of bound compound with the receptor was superimposed with 


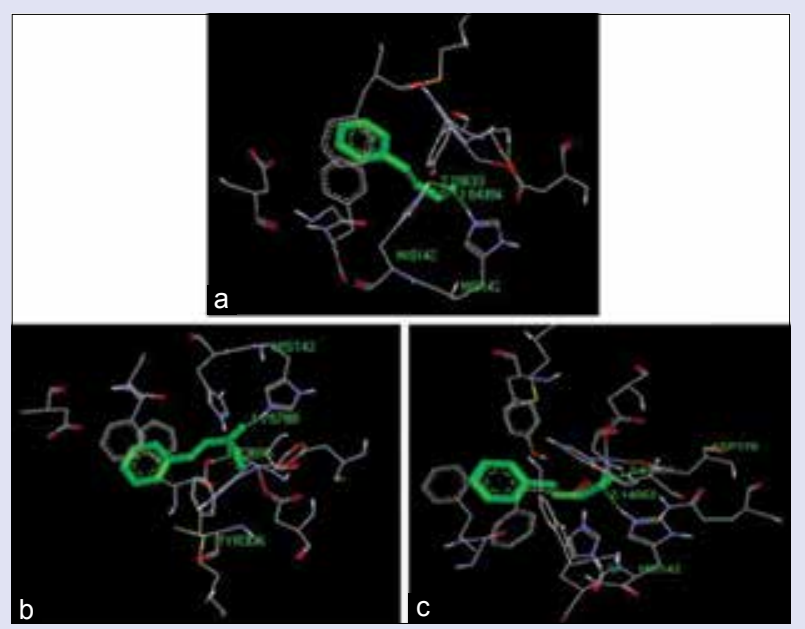

Figure 3: Representation of binding interaction between histone deacetylases family member 8 and (a) cinnamaldehyde, (b) cinnamyl alcohol, and (c) cinnamic acid. Molecular docking simulations indicating interaction of histone deacetylases family member 8 with cinnamaldehyde, cinnamic acid, and cinnamyl alcohol

Table 3: Hydrogen bond interactions of histone deacetylase family member 8 proteins with trichostatin A, cinnamaldehyde, cinnamic acid and cinnamyl alcohol

\begin{tabular}{lllc}
\hline Ligands & Ligand atoms & Residue atoms & Distance $\AA$ \\
\hline TSA & O1 & HIS 142 HE2 & 1.838 \\
& O1 & HIS 143 HE2 & 2.215 \\
\multirow{2}{*}{ CALC } & H & ASP 178 OD2 & 2.091 \\
& H & HIS 142 HE2 & 2.141 \\
CAL & O1 & ASP 178 OD1 & 2.079 \\
& O1 & HIS 142 HE2 & 1.844 \\
CA & O1 & HIS 143 HE2 & 2.186 \\
& O1 & HIS 142 HE2 & 1.758 \\
& TYR 306 HH & 1.737 \\
\hline
\end{tabular}

CAL: Cinnamaldehyde; CA: Cinnamic acid; ACE: Aqueous cinnamon extract; CALC: Cinnamyl alcohol

Table 4: Metal ion Zn-interactions of histone deacetylase family member 8 proteins with trichostatin $\mathrm{A}$, cinnamyl alcohol, cinnamaldehyde, and cinnamic acid

\begin{tabular}{llc}
\hline Ligands & Zn-interactions & Distance $\AA$ \\
\hline TSA & ASP 267 OD2 & 2.0004 \\
& ASP 178 OD2 & 1.9825 \\
& HIS 180 ND1 & 2.0624 \\
CALC & O1, N1 & $2.1301,2.1278$ \\
& HIS 180 ND1 & 2.0624 \\
& ASP 267 OD2 & 2.0004 \\
CAL & ASP 178 OD2 & 1.9825 \\
& O1, N1 & $2.1341,2.1229$ \\
& ASP 178 OD2 & 1.9825 \\
CA & HIS 180 ND1 & 2.0624 \\
& ASP 267 OD2 & 2.0004 \\
& ASP 267 OD2 & 2.0004 \\
& ASP 178 OD2 & 1.9825 \\
& HIS 180 ND1, O1 & $2.0624,1.9243$ \\
\hline
\end{tabular}

CAL: Cinnamaldehyde; CA: Cinnamic acid; ACE: Aqueous cinnamon extract; CALC: Cinnamyl alcohol

original complex downloaded from RCSB PDB (PDB ID: 1T67). ${ }^{[49,52]}$ This was done to calculate RMSD values. Low RMSD $(<2 \AA)$ value of original bound ligand validates the docking procedure. RMSD values were found to be $1.492 \AA$, which indicated that the protocol selected in the docking method was validated.

Molecular docking simulations of HDAC8 proteins with CALC, CA, and CAL were performed using AutoDock 4.2. ${ }^{[48]}$ The accuracy of the AutoDock 4.2 results was confirmed by considering clusters of twenty runs of conformations/orientations with RMSD value 0.0 in addition to the lowest binding free energy and hydrogen bonds between macromolecules. ${ }^{[48]}$ Further, the docked conformations were energetically and statistically validated. Potential binding interactions were observed between ligand and catalytic residues at the active site. The ligand receptor binding interactions are given in Figure 3.

Molecular docking between HDAC8 and TSA showed [Figure 3 and Table 3] high negative binding energy (BE; $-7.18 \mathrm{kcal} / \mathrm{mol}$ ) along with the formation of three strong hydrogen bond interactions with the catalytic residues (His142, His143, and Asp178). Similarly, docking of HDAC8 with CAL, CALC, and CA showed BEs of $-7.18,-5.8$, and $-5.14 \mathrm{kcal} / \mathrm{mol}$, respectively. It was also observed that all the ligands maintained two hydrogen bond interactions within the active site.

The metal ion, $\mathrm{Zn}$ interactions with the ligand, and active site residues were also taken into consideration during the docking study [Table 4].

1T67-TSA complex showed five interactions with zinc ion as well as active site residues Asp267 OD2, Asp178 OD2, His180 ND1, and O1 and N1 of TSA. The proposed inhibitor group compounds, CALC-1T67 complex, showed four interactions with Asp267 OD2, His180 ND1, Asp178 OD2, and O1 of CALC. CAL-1T67 complex showed three metal ion interactions with active site residues, mainly Asp267 OD2, His180 ND1, and Asp267 OD2. CA-1T67 complex showed four Zn ion interactions with Asp267 OD2, Asp178 OD2, His180 ND1, and O1 of CA.

To obtain compounds with good pharmacokinetic properties, absorption, distribution, metabolism, excretion, and toxicity (ADMET) descriptors were calculated using Discovery Studio (DS) [Computer program]. Version Release 4.0. San Diego: Biovia Accelrys Software Inc.; 2015. ${ }^{[54,55]}$ ADMET of the selected compounds was applied to verify whether the molecules were able to cross the blood-brain barrier (BBB) and exhibit better solubility, human intestinal absorption (HIA), and low toxicity. In the present study, main focus was on oral bioavailability, hepatotoxicity, and the capacity to infiltrate the $\mathrm{BBB}$. ADMET properties compute the values of $\mathrm{BBB}$ penetration, solubility, cytochrome P450 (CYP450) 2D6 inhibition, hepatotoxicity, HIA, plasma protein binding (PPB), and an extensive range of ligand toxicity. It has been reported that a drug should not cross BBB level 3. In the present study, TSA showed BBB value of 2 [Table 5] while CAL, CA, and CALC showed values of 1,2 , and 1 , respectively, showing that the values fall within the range that describe a drug candidate. The value of 0 in CYP26 [Table 5] hepatotoxicity for all the compounds, indicated that they exhibited low toxicity. ADMET_ solubility, ADMET_solubility_level, ADMET_EXT_PPB, and ADMET_ Alogp98 of all three cinnamon bioactive compounds were comparable to TSA [Table 5], suggesting that CAL, CA, and CALC could behave as HDAC8 inhibitors.

Frontier orbital (FO) theory states that the shape and symmetries of the highest occupied molecular orbitals (HOMOs) and lowest unoccupied molecular orbitals (LUMOs) are essential to predict the reactivity and the stereochemistry of a compound. ${ }^{[56]}$ For quantum mechanical simulation, DFT is an effective and promising approach of periodic systems, and it offers an accurate elucidation of the electronic and structural behavior of small molecules by calculating the electronic structure of the substance. ${ }^{[57]}$ DFT was performed on CAL, CA, CALC, and TSA to analyze the electronic behavior of the molecules. For this, calculate 
Table 5: Absorption, distribution, metabolism, excretion, and toxicity values of trichostatin A, cinnamic acid, cinnamaldehyde, and cinnamyl alcohol

\begin{tabular}{lccccccc} 
Molecule & $\begin{array}{c}\text { ADMET } \\
\text { solubility }\end{array}$ & $\begin{array}{c}\text { ADMET solubility } \\
\text { level }\end{array}$ & ADMET BBB & $\begin{array}{c}\text { ADMET BBB } \\
\text { level }\end{array}$ & $\begin{array}{c}\text { ADMET extension } \\
\text { of PPB }\end{array}$ & $\begin{array}{c}\text { ADMET_AlogP98 } \\
\text { CYP26_hepato } \\
\text { toxicity }\end{array}$ \\
\hline TSA & -3.226 & 3 & -0.343 & 2 & 0.709 & 3.051 \\
CA & -1.867 & 4 & -0.161 & 2 & 0.797 & 0 \\
CAL & -2.298 & 3 & 0.175 & 1 & 0.885 & 0 \\
CALC & -1.456 & 4 & 0.040 & 1 & -0.457 & 1.927 & 1.693 \\
\hline
\end{tabular}

ADMET: Absorption, distribution, metabolism, excretion, and toxicity; CAL: Cinnamaldehyde; CA: Cinnamic acid; ACE: Aqueous cinnamon extract; CALC: Cinnamyl alcohol; BBB: Blood-brain barrier; EXT: PPB: Plasma protein binding

Table 6: Highest occupied molecular orbital, lowest unoccupied molecular orbital and binding energies of cinnamic acid, cinnamaldehyde, cinnamyl alcohol, and trichostatin A

\begin{tabular}{lccc}
\hline Compounds & BE & HOMO & LUMO \\
\hline CA & -3.874 & -0.223 & -0.107 \\
CAL & -3.667 & -0.195 & -0.105 \\
CALC & -3.876 & -0.195 & -0.054 \\
TSA & -8.746 & -0.171 & -0.073 \\
\hline
\end{tabular}

HOMO: Highest occupied molecular orbital; LUMO: Lowest unoccupied molecular orbital; CAL: Cinnamaldehyde; CA: Cinnamic acid; ACE: Aqueous cinnamon extract; CALC: Cinnamyl alcohol; BE: Binding energy

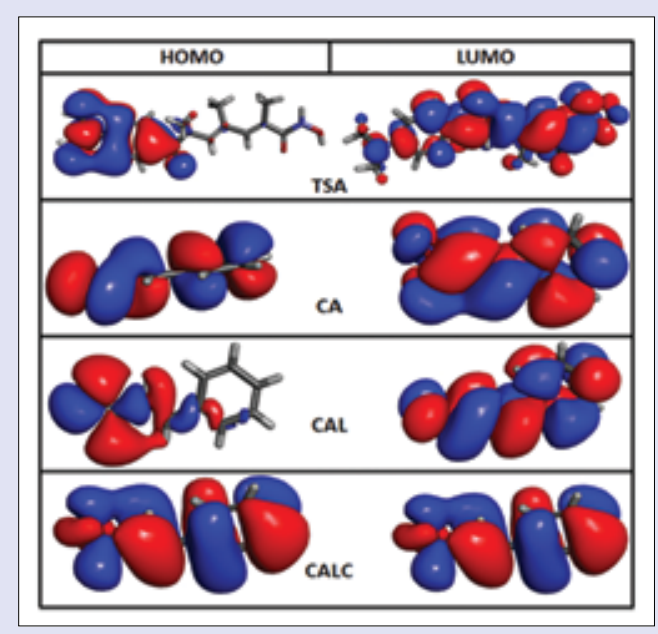

Figure 4: Plots of highest occupied molecular orbitals and lowest unoccupied molecular orbitals of trichostatin A, cinnamic acid, cinnamaldehyde, and cinnamyl alcohol. The quantum chemical descriptors (highest occupied molecular orbitals and lowest unoccupied molecular orbitals) showing map of molecular orbitals indicating the electron exchange and electron transfer ability of trichostatin A, cinnamic acid, cinnamaldehyde, and cinnamyl alcohol

energy (DFT) module of DS was used with B3 LYP algorithm that providing the information, concerning the capability of the molecules to transmission their energies from a HOMO, which can behave as an electron donor, to a LUMO, which can behave as an electron acceptor. ${ }^{[55]}$ The quantum chemical descriptors (HOMO and LUMO) can help to discriminate the reactive or binding sites and substantial stimulus on electronic structure of the molecules. HOMO and LUMO maps have been plotted and depicted in Figure 4. HOMO, LUMO, and BE of CA, CAL, CALC, and TSA are given in Table 6. The molecular orbitals maps indicated that electron exchange and transfer capability of the tested molecules may have a pivotal influence in HDAC8 inhibitory activity. The maps explained that HOMO molecular orbitals were located towards the electron rich area for CAL, CA, and CALC, whereas aromatic rings were also found to be critical for HOMO in TSA, CA, and CALC.
LUMO orbitals were found to be present around the aromatic ring and linear chain of the compounds. The functional groups of CAL, CA, and CALC were found to be important for both the types of orbitals. The results of DFT were fairly consistent with the molecular docking output that illustrated the important contribution of these scaffolds in the key ligand-receptor interactions.

To assess the synthetic feasibility of all the four compounds (TSA, CA, CAL, and CALC), the synthetic accessibility score was measured using SYLVIA v1.4 program. ${ }^{[48,58]}$ This program provides values between 1 and 10 for compounds, suggesting that the synthesis becomes more complex with increasing values. A number of criteria are considered to calculate the synthetic accessibility that include complexity of the molecular structure, complexity of the ring structure, number of stereo centers, resemblance to commercially available compounds, and potential for using powerful synthetic reactions. The outcome of the SYLVIA program indicated that synthetic accessibility score of TSA was 4.81, whereas for CAL, CA, and CALC, the scores were 2.07, 2.09 and 2.05, respectively. Thus, the SYLVIA score for the CAL, CA, and CALC clearly illustrated that selected compounds were easy to synthesize compared to TSA.

\section{CONCLUSION}

We demonstrated HDAC8 inhibitory potential of cinnamon extract and its bioactives. In vitro studies confirmed that ACE significantly inhibited HDAC8 activity compared to the individual bioactives (CAL, CA, and CALC), suggesting that these compounds along with other phenolic compounds might be working synergistically to induce inhibition. Pharmacoinformatics studies revealed that cinnamon bioactives bound to the active site of HDAC8 enzyme in a way similar to that of TSA. The molecular descriptors of cinnamon compounds were successfully correlated with TSA values. The binding interactions and energies were also found to be close to TSA. In case of TSA, His142, His143, and Asp178 amino residues were found to be critical for binding, while His142, His143, Asp178, and Tyr306 were found to be crucial for binding with the cinnamon bioactives. ADMET and DFT calculations were carried out to check the toxicity and reactivity of the tested compounds. All the compounds showed minimum toxicity and values of HOMO and LUMO were comparable to TSA. Finally, synthetic accessibility data indicated that cinnamon bioactives had low synthetic complexity than TSA. Therefore, both experimental and computational studies clearly explained that cinnamon exhibited potential inhibitory activity against HDAC8 enzyme. The data suggested that herbal bioactives could be explored in future for their potential HDAC inhibition to target cancer cells.

\section{Acknowledgments}

We acknowledge our Director, Dr. A.C Mishra, for his constant encouragement and support for completion of this work. 


\section{Financial support and sponsorship}

MA Islam was funded by the University of Pretoria Vice Chancellor's post doctoral and NRF Innovation Post-doctoral fellowship schemes, South Africa, and Choudhari was funded by CSIR Senior Research Fellowship.

\section{Conflicts of interest}

There are no conflicts of interest.

\section{REFERENCES}

1. Delcuve GP, Khan DH, Davie JR. Roles of histone deacetylases in epigenetic regulation: Emerging paradigms from studies with inhibitors. Clin Epigenetics 2012;4:5

2. Singh BN, Zhang G, Hwa YL, Li J, Dowdy SC, Jiang SW. Nonhistone protein acetylation as cancer therapy targets. Expert Rev Anticancer Ther 2010;10:935-54.

3. Yang XJ, Seto E. Lysine acetylation: Codified crosstalk with other posttranslational modifications. Mol Cell 2008;31:449-61.

4. Fischle W, Dequiedt F, Hendzel MJ, Guenther MG, Lazar MA, Voelter W, et al. Enzymatic activity associated with class II HDACs is dependent on a multiprotein complex containing n3 and SMRT/N-CoR. Mol Cell 2002;9:45-57.

5. Jones P, Altamura S, De Francesco R, Gallinari P, Lahm A, Neddermann P, et al. Probing the elusive catalytic activity of vertebrate class Ila histone deacetylases. Bioorg Med Chem Lett 2008;18:1814-9.

6. Lahm A, Paolini C, Pallaoro M, Nardi MC, Jones P, Neddermann P, et al. Unraveling the hidden catalytic activity of vertebrate class Ila histone deacetylases. Proc Natl Acad Sci U S A 2007;104:17335-40.

7. Bonda DJ, Lee HG, Camins A, Pallàs M, Casadesus G, Smith MA, et al. The sirtuin pathway in ageing and Alzheimer disease: Mechanistic and therapeutic considerations. Lancet Neurol 2011;10:275-9.

8. Houtkooper $\mathrm{RH}$, Pirinen $\mathrm{E}$, Auwerx J. Sirtuins as regulators of metabolism and healthspan. Nat Rev Mol Cell Biol 2012;13:225-38.

9. Hu J, Jing H, Lin H. Sirtuin inhibitors as anticancer agents. Future Med Chem 2014;6:945-66.

10. Borradaile NM, Pickering JG. NAD(+), sirtuins, and cardiovascular disease. Curr Pharm Des 2009;15:110-7

11. Abbas A, Gupta S. The role of histone deacetylases in prostate cancer. Epigenetics 2008;3:300-9.

12. Vannini A, Volpari C, Filocamo G, Casavola EC, Brunetti M, Renzoni D, et al. Crystal structure of a eukaryotic zinc-dependent histone deacetylase, human HDAC8, complexed with a hydroxamic acid inhibitor. Proc Natl Acad Sci U S A 2004;101:15064-9.

13. Rettig I, Koeneke E, Trippel F, Mueller WC, Burhenne J, Kopp-Schneider A, et al. Selective inhibition of HDAC8 decreases neuroblastoma growth in vitro and in vivo and enhances retinoic acid-mediated differentiation. Cell Death Dis 2015;6:e1657.

14. Somoza JR, Skene RJ, Katz BA, Mol C, Ho JD, Jennings AJ, et al. Structural snapshots of human HDAC8 provide insights into the class I histone deacetylases. Structure 2004;12:1325-34.

15. Kim HJ, Bae SC. Histone deacetylase inhibitors: Molecular mechanisms of action and clinical trials as anti-cancer drugs. Am J Transl Res 2011;3:166-79.

16. Thurn KT, Thomas S, Moore A, Munster PN. Rational therapeutic combinations with histone deacetylase inhibitors for the treatment of cancer. Future Oncol 2011;7:263-83.

17. Senawong T, Misuna S, Khaopha S, Nuchadomrong S, Sawatsitang P, Phaosiri C, et al. Histone deacetylase (HDAC) inhibitory and antiproliferative activities of phenolic-rich extracts derived from the rhizome of Hydnophytum formicarum Jack.: Sinapinic acid acts as HDAC inhibitor. BMC Complement Altern Med 2013;13:232.

18. Duthie GG, Duthie SJ, Kyle JA. Plant polyphenols in cancer and heart disease: Implications as nutritional antioxidants. Nutr Res Rev 2000;13:79-106.

19. Yang CS, Landau JM, Huang MT, Newmark HL. Inhibition of carcinogenesis by dietary polyphenolic compounds. Annu Rev Nutr 2001;21:381-406.

20. Ramchoun M, Harnafi H, Alem C, Benlyas M, Elrhaffari L, Amrani S. Study on antioxidant and hypolipidemic effects of polyphenol-rich extracts from Thymus vulgaris and Lavendula multifida. Pharmacogn Res 2009;1:106-12.

21. Link A, Balaguer F, Goel A. Cancer chemoprevention by dietary polyphenols: Promising role for epigenetics. Biochem Pharmacol 2010;80:1771-92.

22. Radhakrishnan DK, Chacko SM, Bhaskara PK, Sandya S, Govindan NK. Polyphenolic extract from coconut kernel modulates apoptotic genes, reactive oxygen species production, and prevents proliferation of human colon cancer cell line. Int J Clin Exp Physiol 2016;3:113.

23. Rajendran P, Ho E, Williams DE, Dashwood RH. Dietary phytochemicals, HDAC inhibition, and DNA damage/repair defects in cancer cells. Clin Epigenetics 2011;3:4.

24. Shan B, Cai YZ, Sun M, Corke H. Antioxidant capacity of 26 spice extracts and characterization of their phenolic constituents. J Agric Food Chem 2005;53:7749-59.

25. Mathew S, Abraham TE. Studies on the antioxidant activities of cinnamon (Cinnamomum verum) bark extracts, through various in vitro models. Food Chem 2006;94:520-8.

26. Lobo V, Patil A, Phatak A, Chandra N. Free radicals, antioxidants and functional foods: Impact on human health. Pharmacogn Rev 2010;4:118-26.

27. Chao LK, Hua KF, Hsu HY, Cheng SS, Liu JY, Chang ST. Study on the antiinflammatory activity of essential oil from leaves of Cinnamomum osmophloeum. J Agric Food Chem 2005;53:7274-8.

28. Tung YT, Chua MT, Wang SY, Chang ST. Anti-inflammation activities of essential oil and its constituents from indigenous cinnamon (Cinnamomum osmophloeum) twigs. Bioresour Technol 2008:99:3908-13.

29. Matan N, Rimkeeree H, Mawson AJ, Chompreeda P, Haruthaithanasan V, Parker M Antimicrobial activity of cinnamon and clove oils under modified atmosphere conditions. Int J Food Microbiol 2006;107:180-5.

30. El-Baroty GS, Abd El-Baky HH, Farag RS, Saleh MA. Characterization of antioxidant and antimicrobial compounds of cinnamon and ginger essential oils. Afr $\mathrm{J}$ Biochem Res 2010;4:167-74

31. Noudeh GD, Sharififar F, Noodeh AD, Moshafi MH, Afzadi MA, Behravan E, et al. Antitumor and antibacterial activity of four fractions from Heracleum persicum Desf. and Cinnamomum zeylanicum Blume. J Med Plants Res 2010;4:2176-80

32. Gupta C, Garg AP, Uniyal RC, Kumari A. Comparison of antimicrobial activities of clove oil \& its extract on some food borne microbes. Afr J Microbiol Res 2009;7:247-51.

33. Kurokawa M, Kumeda CA, Yamamura J, Kamiyama T, Shiraki K. Antipyretic activity of cinnamyl derivatives and related compounds in influenza virus-infected mice. Eur $J$ Pharmacol 1998:348:45-51.

34. Akira T, Tanaka S, Tabata M. Pharmacological studies on the antiulcerogenic activity of Chinese cinnamon. Planta Med 1986;6:440-3.

35. Rezq AA, Elmallh MM. Anti-ulcer effect of cinnamon and chamomile aqueous extracts in rat models. J Am Sci 2010;6:209-16.

36. Kim SH, Hyun SH, Choung SY. Anti-diabetic effect of cinnamon extract on blood glucose in db/db mice. J Ethnopharmacol 2006;104:119-23.

37. Verspohl EJ, Bauer K, Neddermann E. Antidiabetic effect of Cinnamomum cassia and Cinnamomum zeylanicum in vivo and in vitro. Phytother Res 2005;19:203-6.

38. Kim SH, Choung SY. Antihyperglycemic and antihyperlipidemic action of Cinnamomi Cassiae (Cinnamon bark) extract in C57BL/Ks db/db mice. Arch Pharm Res 2010;33:325-33

39. Rind NA, Dahot MU, Malik SA, Kumar M. Antidiabetic effect of cinnamon (Cinnamonum zeylanicum) used to control diabetes mellitus in alloxan induced Diabetic male rabbits. Pak J Biotechnol 2010;7:81-90.

40. Mohamed Sham Shihabudeen H, Hansi Priscilla D, Thirumurugan K. Cinnamon extract inhibits a-glucosidase activity and dampens postprandial glucose excursion in diabetic rats. Nutr Metab (Lond) 2011;8:46.

41. Koppikar SJ, Choudhari AS, Suryavanshi SA, Kumari S, Chattopadhyay S, Kaul-Ghanekar R Aqueous cinnamon extract (ACE-c) from the bark of Cinnamomum cassia causes apoptosis in human cervical cancer cell line ( $\mathrm{SiH}$ ) through loss of mitochondrial membrane potential. BMC Cancer 2010;10:210

42. Kamei T, Kumano H, Iwata K, Nariai Y, Matsumoto T. The effect of a traditional Chinese prescription for a case of lung carcinoma. J Altern Complement Med 2000;6:557-9.

43. Ai G, Tian C, Deng D, Fida G, Chen H, Ma Y, et al. A combination of 2D similarity search, pharmacophore, and molecular docking techniques for the identification of vascular endothelial growth factor receptor 2 inhibitors. Anticancer Drugs 2015;26:399 409.

44. Giaginis C, Tsantili-Kakoulidou A, Theocharis S. Applying quantitative structure-activity relationship (QSAR) methodology for modeling postmortem redistribution of benzodiazepines and tricyclic antidepressants. J Anal Toxicol 2014;38:242-8.

45. Hayashi Y, Sugiyama H, Suganami A, Higashi K, Kashiwagi K, Igarashi K, et al. Prediction of the interaction between spermidine and the $\mathrm{G} G$ mismatch containing acceptor stem in tRNA (lle): Molecular modeling, density functional theory, and molecular dynamics study. Biochem Biophys Res Commun 2013:441:999 1004. 
46. Bautista Aguilera OM, Esteban G, Chioua M, Nikolic K, Agbaba D, Moraleda I, et al. Multipotent cholinesterase/monoamine oxidase inhibitors for the treatment of Alzheimer's disease: Design, synthesis, biochemical evaluation, ADMET, molecular modeling, and QSAR analysis of novel donepezil pyridyl hybrids. Drug Des Devel Ther 2014;8:1893 910

47. Bautista-Aguilera OM, Esteban G, Chioua M, Nikolic K, Agbaba D, Moraleda I, et al. Multipotent cholinesterase/monoamine oxidase inhibitors for the treatment of Alzheimer's disease: Design, synthesis, biochemical evaluation, ADMET, molecular modeling, and QSAR analysis of novel donepezil-pyridyl hybrids. Drug Des Devel Ther 2014;8:1893-910.

48. Morris GM, Huey R, Lindstrom W, Sanner MF, Belew RK, Goodsell DS, et al. AutoDock4 and AutoDockTools4: Automated docking with selective receptor flexibility. J Comput Chem 2009;30:278591.

49. Bolden JE, Shi W, Jankowski K, Kan CY, Cluse L, Martin BP, et al. HDAC inhibitors induce tumor cell selective pro apoptotic transcriptional responses. Cell Death Dis 2013;4:e519.

50. Verma A. Lead finding from Phyllanthus debelis with hepatoprotective potentials. Asian Pac J Trop Biomed 2012;2:1735-7.

51. Lipinski CA, Lombardo F, Dominy BW, Feeney PJ. Experimental and computational approaches to estimate solubility and permeability in drug discovery and development settings. Adv Drug Deliv Rev 2001;46:3-26

52. Mottamal M, Zheng S, Huang TL, Wang G. Histone deacetylase inhibitors in clinical studies as templates for new anticancer agents. Molecules 2015;20:3898-941.
53. Priya R, Sumitha R, Doss CG, Rajasekaran C, Babu S, Seenivasan R, et al. Molecular docking and molecular dynamics to identify a novel human immunodeficiency virus inhibitor from alkaloids of Toddalia asiatica. Pharmacogn Mag 2015;11 Suppl 3:S414-22.

54. Taha MO, Habash M, Al-Hadidi Z, Al-Bakri A, Younis K, Sisan S. Docking-based comparative intermolecular contacts analysis as new 3-D QSAR concept for validating docking studies and in silico screening: NMT and GP inhibitors as case studies. J Chem Inf Model 2011;51:647-69

55. Discovery Studio Modeling Environment [Computer program]. Version Release 3.1. San Diego: Accelrys Software Inc.; 2012.

56. Eddy NO, Ebenso EE, Ibok UJ, Akpan EE. Experimental and computational chemistry studies on the inhibition ofthecorrosion of mildsteelinH2SO4by (2s, 5s,6r)-6-(2-(aminomethyl)-5-(3-(2-chlorophenyl) isoxazol-5-yl) benzamido)-3,3-dimethyl-7-oxo-4-thia-1-azabicyclo[3.2.0] heptane-2-carboxylic acid. Int J Electrochem Sci 2011;6:4296-315.

57. Sakkiah S, Lee KW. Pharmacophore-based virtual screening and density functional theory approach to identifying novel butyrylcholinesterase inhibitors. Acta Pharmacol Sin 2012;33:964-78

58. Boda K, Seidel T, Gasteiger J. Structure and reaction based evaluation of synthetic accessibility. J Comput Aided Mol Des 2007;21:311-25.

59. Morris GM, Huey R, Lindstrom W, Sanner MF, Belew RK, Goodsell DS, et al. AutoDock4 and AutoDockTools4: Automated docking with selective receptor flexibility. J Comput Chem 2009:30:2785-91. 